\title{
Métodos para medir la calidad del servicio turístico en empresas de alojamiento
}

\section{Methods to measure the quality of the tourist service in accommodation companies}

\author{
MSc. Diego Bonilla Jurado ${ }^{1}$ \\ dbonilla@ueb.edu.ec \\ MSc. Aura Guerrero Luzuriaga \\ aguerrero@ucacue.edu.ec \\ MSc. Oswaldo Ernesto López Bravo \\ olopez@ueb.edu.ec
}

Recibido: 1/04/2018, Aceptado: 1/06/2018

\begin{abstract}
RESUMEN
Los cambios en las empresas de alojamiento son causados principalmente por la creciente globalización y la creciente competencia. Cadenas hoteleras globales dominan la industria a pesar del creciente número de pequeños y medianos hoteles y otras capacidades de alojamiento. Esta investigación aborda los antecedentes y la estructura de la calidad del servicio en el contexto de empresas de alojamiento, además de los métodos comúnmente utilizados para medir su gestión en relación a la calidad del servicio otorgado hacia el cliente. Entre los métodos frecuentemente utilizados se encontraron el modelo SERVQUAL, HOTELQUAL, LODGSERV, SERVPERF y otras técnicas que ayudan a medir la satisfacción del cliente pudiendo ser en este caso diseño de cuestionarios, sistemas de sugerencias y reclamaciones y la técnica del Mistery Shoppers. Se realizó una revisión de literatura, que engloba artículos científicos publicados en diferentes revistas a nivel mundial. Se procesaron un total de 29 artículos, distribuidos de la siguiente manera: 19 artículos científicos, 6 libros y 4 proyectos de investigación que abordaron temas como la calidad del servicio y métodos de medición en el sector hotelero. Una vez revisada la bibliografía correspondiente se determinó que el método SERVQUAL es el más utilizado dentro de la medición de la calidad de servicio comparando las perspectivas vs las expectativas.
\end{abstract}

\footnotetext{
1 Universidad Estatal de Bolívar, Ecuador

2 Universidad Católica de Cuenca, Ecuador

3 Universidad Estatal de Bolívar, Ecuador
} 


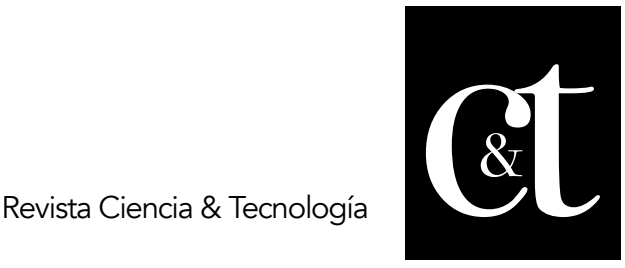

No. 19, 31 de julio de 2018

ISSN impreso: 1390 - 6321

Palabras clave: Calidad del servicio, empresas de alojamiento, HOTELQUAL, métodos de medición, SERVQUAL

\begin{abstract}
Changes in hosting companies are mainly caused by increasing globalization and increasing competition. Global hotel chains dominate the industry despite the growing number of small and medium-sized hotels and other accommodation capabilities. This research addresses the background and structure of service quality in the context of hosting companies, in addition to the methods commonly used to measure their management in relation to the quality of the service provided to the client. Among the methods frequently used were the SERVQUAL, HOTELQUAL, LODGSERV, SERVPERF and other techniques that help measure customer satisfaction, in this case designing questionnaires, suggestions and claims systems and the Mistery Shoppers technique. A literature review was carried out, which includes scientific articles published in different journals worldwide. A total of 29 articles were processed, distributed as follows: 19 scientific articles, 6 books and 4 research projects that addressed issues such as quality of service and measurement methods in the hotel sector. Once the corresponding bibliography was reviewed, it was determined that the SERVQUAL method is the most used in the measurement of quality of service, comparing perspectives vs. expectations.
\end{abstract}

Keywords: Service quality, lodging firms, HOTELQUAL, measurement methods, SERVQUAL

\title{
Introducción
}

En vista de la creciente competencia, el sector hotelero se encuentra en búsqueda de alternativas nuevas que permitan crear ventajas competitivas. Por lo que, se está poniendo una gran cantidad de esfuerzo en la selección de los mejores métodos y herramientas para medir el desarrollo de la calidad del servicio. En relación a la medición de la calidad del servicio en empresas de alojamiento, se registran numerosas metodologías, sin embargo, la más utilizada constituye el método SERVQUAL, el mismo que es aplicado como una herramienta para la comprensión de los factores que afectan a la calidad del servicio en las empresas de alojamiento, desde la perspectiva de los clientes. Los resultados de estos estudios han entregado contribuciones en relación con la comprensión de la estructura tridimensional de la calidad del servicio. Varios estudios han postulado que la calidad del servicio (SERVQUAL) es multidimensional, en esencia, para medir la calidad del servicio. En un estudio realizado por [1] sobre la calidad del servicio de los hoteles en Australia se determinó que los siete factores se desarrollaron a partir SERVQUAL, que cubre el estilo y la comodidad, calidad de la habitación, personal de calidad, ofertas especiales, servicio rápido, servicio personalizado, servicio rápido y calidad de los alimentos y bebidas. Por otro lado, en el estudio realizado por [2] cuya investigación se centra en la medición de la calidad del servicio de hoteles en Turquía a través del análisis de la expectativa y la percepción real de la calidad del servicio basado en 29 indicadores característicos, que se aplicaron a partir del modelo SERVQUAL, y el factor más importante que refleja las necesidades generales de medición de la calidad del servicio eran los servicios tangibles. Cabe señalar que, también se registran, diversas investigaciones donde se ha indicado y explicado que las características de los factores de calidad de servicio y las 


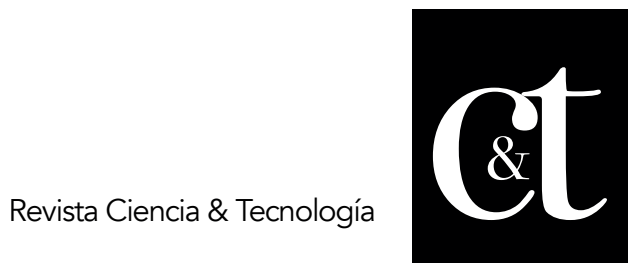

No. 19, 31 de julio de 2018

ISSN impreso: 1390 - 6321

prioridades de servicio, desde la perspectiva de los clientes [3].

Es decir, a partir de la perspectiva de los clientes, las brechas entre las expectativas y la percepción reflejan explícitamente la calidad del servicio de los hoteles. Una brecha positiva $(+)$ significa que la calidad del servicio de los hoteles es buena y los hoteles ofrecen servicios que son mejores de lo esperado; por otro lado, una brecha negativa (-) significa que la calidad del servicio de los hoteles es mala y los hoteles entregaron peores servicios de los esperaba; por el contrario, una brecha de cero (0) significa que la calidad del servicio de los hoteles satisface las necesidades de los clientes [4].

El modelo SERVQUAL, es el modelo más popular para medir la satisfacción del cliente en casi todos los servicios industriales. El modelo fundamenta en la evaluación del cliente de calidad del servicio, que no es otra cosa que una comparación de lo esperado y el valor obtenido [5].

La importancia de ofrecer un servicio de calidad ha sido objeto de muchos estudios, y de ahí el interés en el desarrollo de modelos que permitan su medida; como el desarrollado por [6]: SERVQUAL (Servicio de Calidad). Los autores proponen una herramienta delineado por un cuestionario, para medir la calidad de un servicio con la escala SERVQUAL, fundamentada principalmente en las diferencias entre la percepción y las expectativas de los clientes sobre las dimensiones más significativas de un servicio particular. Los resultados cuantitativos que ofrece el modelo pueden ser utilizado como patrón para determinar los costos de la "mala calidad" de los servicios, y el valor de las inversiones que se pueden hacer (económicos y humanos) para mejorar la calidad [7].

De forma general se acepta que la calidad del servicio constituye el antecedente de la satisfacción del cliente, mientras que la satisfacción del cliente es antecedente de la lealtad del cliente. Bajo este contexto se puede argumentar que la calidad de servicio percibida deriva del encuentro del servicio individual entre el cliente y el proveedor de los servicios, durante el cual el cliente evalúa la calidad y experimenta la satisfacción o insatisfacción [1]. Cada experiencia de servicio se compone de una serie de encuentros individuales durante la cual el cliente va a realizar estas evaluaciones [8].

Las expectativas del servicio se encuentran influidas por las señales intrínsecas y extrínsecas relacionadas con una experiencia particular y por una perspectiva global construido a partir de experiencias previas y otras fuentes de información. La experiencia de los servicios será evaluada por los consumidores sobre la base de sus expectativas a priori y se utiliza para evaluar la calidad, para determinar la satisfacción y formar expectativas sobre las experiencias de consumo futuro [1].

\section{Desarrollo}

Calidad en los servicios

La calidad representa un término que se considera indicativo de un alto nivel de satisfacción de los clientes y se refiere a los factores que caracterizan a un producto o servicio. La calidad del servicio se podría considerar como un compuesto de múltiples atributos. No sólo se compone de atributos tangibles, sino también de intangibles y atributos subjetivos tales como la comodidad, seguridad y satisfacción, 
que son difíciles de medir con precisión [9]. Teniendo en cuenta las características de los servicios (intangibilidad, inseparabilidad, heterogeneidad y perecedero), se hace difícil medir su calidad. Bajo este contexto, se puede describir la calidad del servicio como la capacidad de satisfacer las necesidades del cliente. Asimismo, La evaluación de la calidad percibida se realiza por el cliente durante o después del proceso de prestación de servicios; y se determina comparando la calidad percibida vs la esperada por el cliente [10].

En lo que hace mención a la calidad en los servicios y la percepción que tienen los clientes frente a la misma, se puede hablar de valores, necesidades y expectativas de los consumidores que se reconocen. Bajo esta perspectiva, la percepción podría ser entendida mejor a partir de los elementos que la conforman: el estímulo, los receptores sensoriales y finalmente, la sensación [9]. A continuación, en la figura 1 se muestra la relación entre las expectativas y las percepciones de la calidad en los servicios.

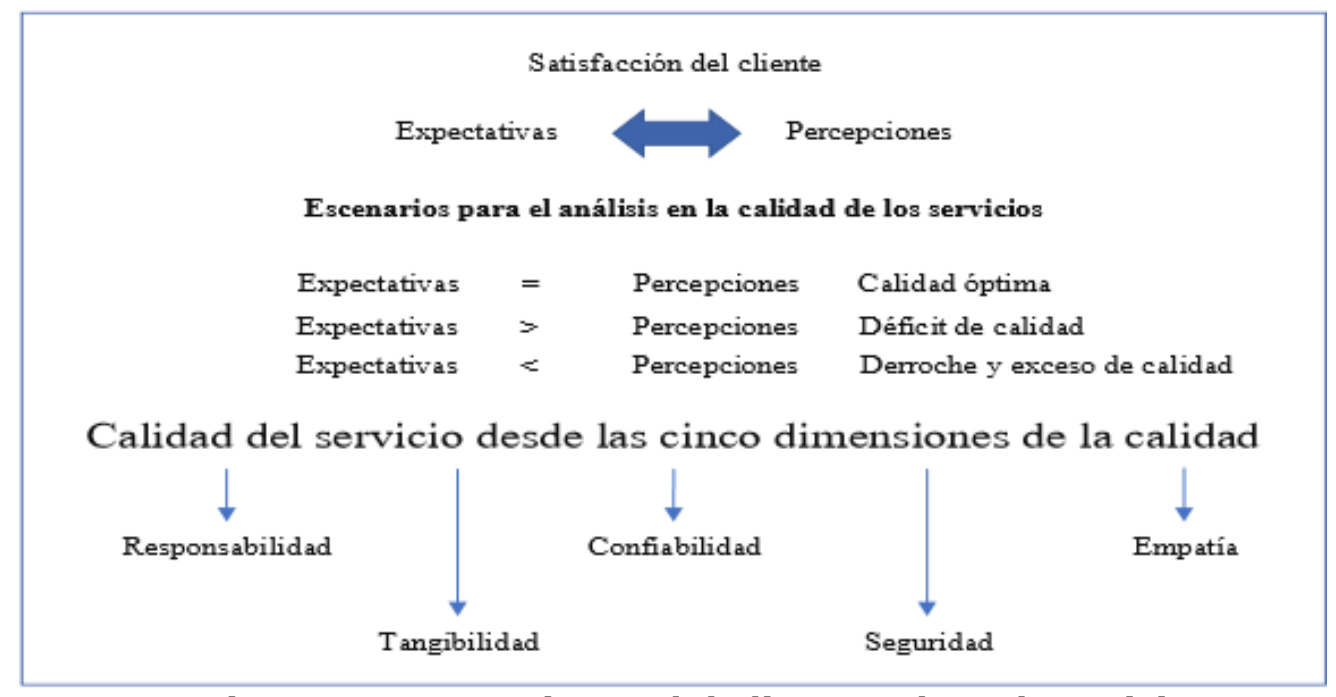

Figura 1. Percepciones del cliente sobre el servicio

Es necesario realizar mediciones periódicas de la calidad de los servicios; para ello se deben crear instrumentos que ayuden a los gerentes a realizar dichas mediciones, dado que lo que no se puede medir no es posible controlar [11].

La calidad del servicio es la percepción que tienen los usuarios sobre la correspondencia entre el desempeño y las expectativas de la experiencia de servicio. La calidad de servicio constituye la diferencia o discrepancia que existe entre los deseos de los clientes y la percepción del conjunto de elementos cualitativos y cuantitativos de un producto o servicio principal [12].

Se denomina calidad del servicio a la dirección y grado de discrepancia entre la percepción del cliente y sus expectativas, en términos de las dimensiones de la calidad del servicio que pueden afectar el comportamiento futuro de los consumidores [13]. 
Determinantes de la calidad del servicio

Para cada tipo de servicio, no se puede identificar un conjunto específico de determinantes de calidad. Sin embargo, diferentes estudios [6] han tratado de definir un conjunto genérico de determinantes que se aplica a todos los tipos de servicio. Con base a estos estudios, a continuación, se describen los determinantes de la calidad que en su mayoría son aplicados dentro de los diferentes servicios que son comercializados dentro de mercado:

Tangibles: Incluyen el estado de facilitar bienes, es decir, la condición física de los edificios y el medio ambiente, el aspecto del personal, y el estado del equipo.

Personalización: La disposición y capacidad para ajustar el servicio para satisfacer las necesidades del cliente.

Acceso: La facilidad de cercanía y contacto.

Comunicación: Mantener informados a los clientes sobre el servicio en un idioma que puedan entender y escuchar.

Cortesía: El respeto, la consideración y amabilidad se muestra a los clientes por el personal de contacto.

Competencia: Los empleados deben poseer las habilidades necesarias, el conocimiento y la información para realizar el servicio con eficacia.

Credibilidad: la medida en que se cree que el servicio de confianza. El nombre del proveedor de servicios y la reputación, y los rasgos personales de los empleados de primera línea, todo ello contribuye a la credibilidad.

Confiabilidad: la capacidad de proporcionar el servicio prometido a tiempo, de forma precisa y fiable.

Sensibilidad: la capacidad de hacer frente eficazmente a las quejas y la rapidez del servicio.

Costo: constituye el precio pagado por el servicio. Aunque el precio es un criterio competitivo que tiene una fuerte influencia en el posicionamiento.

Cada tipo de servicio puede tener diferentes determinantes que pudieran ser considerados como críticos para la empresa. La percepción de la calidad y la posterior evaluación del servicio se dan respecto a los determinantes que se consideran más significativas para el cliente en cada momento de la verdad, es decir donde ocurre la interacción. En definitiva, la calidad del servicio se ve directamente influenciada por el servicio esperado y el servicio percibido. Por lo que, si los servicios son recibidos como eran de esperar, la calidad del servicio será satisfactoria, además, si los servicios recibidos superan las expectativas de los clientes éstos estarán encantados, y percibirán la calidad del servicio como excelente [10]. 
Calidad del servicio turístico y percepción del cliente

Uno de los objetivos fundamentales de la calidad es lograr la satisfacción del cliente. Como consecuencia de los cambios en los gustos y necesidades de los turistas, la incorporación de nuevas tecnologías, la intensificación de la competencia y la preocupación por el deterioro del medio ambiente, entre otros factores, la oferta de un producto rígido y masivo ya no satisface plenamente la demanda. Los clientes actualmente precisan un interés creciente por la prestación de un servicio individualizado y de calidad [15]. A continuación, dentro de la figura 2 se esquematiza las etapas para la formación de la calidad percibida.

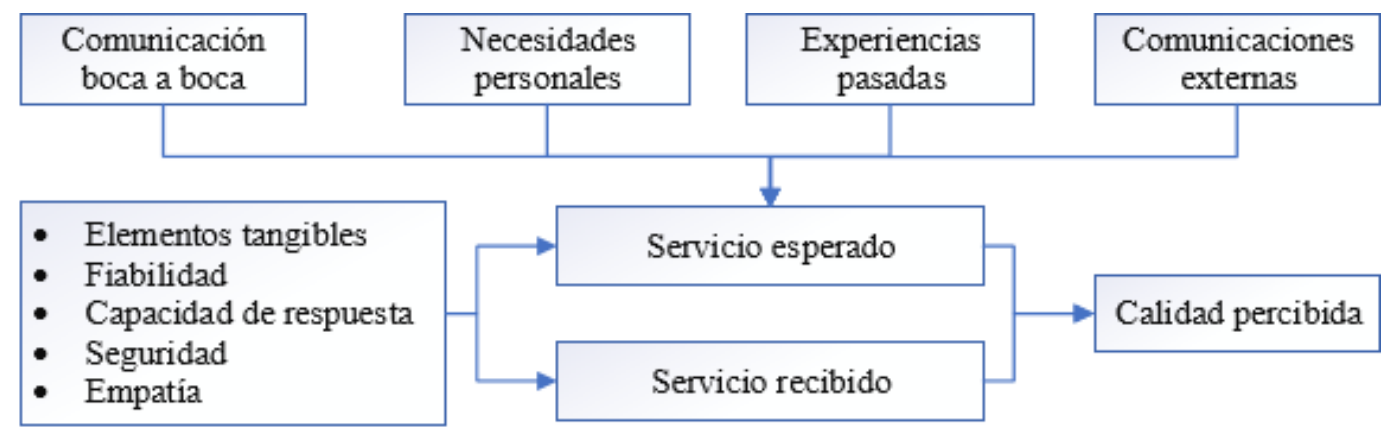

Figura 2. Formación de la calidad percibida

Un cliente satisfecho proclamará las excelentes cualidades del servicio prestado a través del boca a boca, una fuente de comunicación que goza de una gran credibilidad, al estar basada en la experiencia del que emite la opinión, y que además no obtiene ningún beneficio de la misma cuando un cliente se marcha insatisfecho de un establecimiento turístico, no solo corre el riesgo de perderlo, sino que la situación aún puede agravarse a consecuencia de los efectos de la comunicación de su insatisfacción a otras personas, pudiendo en el peor de los escenarios perder un número grande de clientes potenciales que difícilmente son cuantificados [16].

Así pues, entre las expectativas (servicio esperado) y las percepciones (servicio recibido) se puede producir tres escenarios distintos que cada una de ellas puede tener reacciones diferentes en los clientes:

Percepción $=$ Expectativas $=$ Cliente satisfecho $=$ Buena calidad

Significa que el cliente se encuentra satisfecho por lo que, en el futuro éste regresará a consumir el producto o servicio.

Percepción $<$ Expectativas $=$ Cliente insatisfecho $=$ Mala calidad

Significa un posible cliente perdido, además de los clientes potenciales perdidos por el boca a boca.

Percepción $>$ Expectativas $=$ Cliente muy satisfecho $=$ cliente fidelizado

Seguramente este cliente volverá a consumir el producto o servicio y además se lo recomendará a todos sus conocidos.

Con base a estos escenarios, los únicos criterios que son valederos dentro de la evaluación de la calidad de un servicio son los que ponen de manifiesto los clientes, 
por lo tanto, resulta necesario que las empresas turísticas analicen aspectos tales como: cuáles son los factores que más influyen en su valoración cuando consumen el producto turístico y qué esperan éstos de ellas, con la finalidad de que la calidad percibida por el cliente sea superior a lo que en principio éste esperada. Sin embargo, realizar esta medición no resulta fácil, dada las complicaciones en la recogida de información. Para cumplir el objetivo de medir la calidad del servicio se han creado algunos modelos: el principal es el modelo SERVQUAL, que se puede aplicar a cualquier tipo de servicio. Otros modelos similares pero desarrollados de forma específica para el sector turístico son los siguientes: LODGSERV, para la medición de la calidad de servicios en hoteles; HOTELQUAL, para servicios de alojamiento; SERVPERF, que pretende medir la calidad percibida por los clientes [17].

\section{Modelo SERVQUAL}

Medir los productos y servicios implica medir o calibrar la satisfacción del cliente, a partir de aquello, el sistema más aceptado dentro de las empresas de alojamiento y turismo constituye el modelo SERVQUAL, cuya metodología se desarrolla bajo cinco vertientes que caracterizan a un servicio y las cuales se encuentran representadas dentro de un cuestionario estructurado por un total de 22 preguntas o ítems. Los datos obtenidos posterior a su aplicación permiten identificar y cuantificar los aspectos más significativos que ayudan a determinar el nivel de satisfacción que experimentan los diferentes clientes posteriores a la utilización del servicio. En consecuencia, la medición del grado de satisfacción de los clientes proporciona la medición de la calidad de un servicio [18].

La aplicación del modelo SERVQUAL consiste en evaluar las expectativas que tiene el cliente sobre el servicio que va a recibir y la evaluación de las percepciones del servicio que ha recibido. Posterior a esta actividad, se determinan las discrepancias resultantes de cada una de las dimensiones, es decir, lo que debe mejorar la empresa en relación al servicio ofrecido [19].

Bajo este contexto, este modelo ha sido planteado para intentar dar respuesta a una de las principales dificultades que presenta la aplicación de la calidad total a las empresas de servicios, como es la medición de sus resultados.

Este modelo se fundamenta en la consideración de la calidad como una expectativa y su medición como el resultado de comparar lo que el cliente cree que la empresa debe ofrecer (expectativas), con la percepción del desempeño que se tiene del servicio recibido (percepciones) [14].

La evaluación del servicio en este modelo se identifican cinco dimensiones o criterios relevantes del mismo, siendo estos: elementos tangibles, fiabilidad, capacidad de respuesta, seguridad y empatía. A continuación, dentro de los siguientes epígrafes se realiza una descripción breve de cada uno de estos factores.

- Elementos tangibles: hace referencia a la apariencia y funcionalidad del establecimiento [20]; es decir, la apariencia de las instalaciones físicas, equipos, personal de contacto y material de comunicación [21]

- Fiabilidad: refleja la medida en que el establecimiento y sus empleados prestan el servicio de forma correcta y consistente [20]; es decir, constituye 


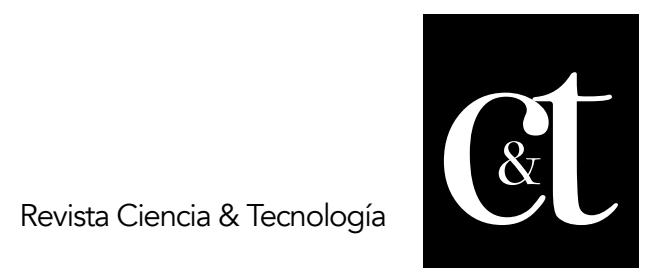

No. 19, 31 de julio de 2018

ISSN impreso: 1390 - 6321

la habilidad para realizar el servicio prometido de forma cuidadosa [21].

- Capacidad de respuesta: constituye la habilidad de los empleados para proporcionar un servicio que responda de manera rápida y eficiente a los diferentes requerimientos que realicen los clientes [20]; es decir, la disposición para ayudar a los usuarios y proporcionar un servicio rápido [21].

- Seguridad: se refiere al conocimiento y la atención mostrados por los empleados y las habilidades de que disponen para inspirar confianza y credibilidad. En esta dimensión deben darse conjuntamente competencia y cortesía [17].

- Empatía: representa la accesibilidad y la manera en que la dirección y los empleados se preocupan de las necesidades de los individuos [20]; es decir, la atención individualizada que ofrece la empresa a sus clientes. Ponerse en el lugar del cliente para tratar de identificar sus necesidades [22].

Modelo HOTELQUAL

El objetivo de este modelo fue elaborar una escala para medir la calidad percibida por clientes de servicios de alojamiento. A esta escala se la denominó HOTELQUAL, misma que fue traba-jada en dos fases. En una primera fase, los autores partieron de la revisión de la literatura, tomaron como referencia y base el modelo SERVQUAL y realizaron un cuestionario adaptado al sector hotelero. Por otro lado, la segunda fase estuvo estructurado por la aplicación del cuestionario a una muestra representativa de usuarios de hoteles tras lo cual pasó a validarse la nueva escala de medida. Los resultados obtenidos dentro del levantamiento de información muestran que los cinco atributos del modelo SERVQUAL no son válidos, dado que el estudio, posterior al análisis factorial, indica una agrupación en tres atributos, donde se destacan: valoración del personal que presta el servicio, valoración de las instalaciones del hotel y percepción sobre el funcionamiento y organización de los servicios que presta el hotel [23].

Asimismo, este modelo toma como referencia las preguntas o ítems propuestos dentro de la escala SERVQUAL y los adapta a los servicios de alojamiento, tomando en consideración tres factores indispensables: la evaluación del personal, la evaluación de las instalaciones y su funcionamiento y la organización del servicio [24].

Bajo este criterio, el modelo HOTELQUAL se encuentra estructurado por un total de 20 preguntas, algunas de ellas, coinciden con los del modelo SERVQUAL, sin embargo, otros se han adaptado al servicio de alojamiento, en donde la calidad es considerada en términos parecidos al SERVQUAL, de modo que, también se registran discrepancias entre expectativas y rendimiento (ver tabla 1) [25]. 
Tabla 1. Discrepancias entre expectativas y rendimiento

\begin{tabular}{|l|l|}
\hline Expectativas & Rendimiento \\
\hline $\begin{array}{l}\text { Dependencias y equipamiento del } \\
\text { edificio deben estar bien conservados } \\
\text { (habitaciones, ascensores, pasillos } \\
\text { entre otros) }\end{array}$ & $\begin{array}{l}\text { Dependencias y equipamiento del } \\
\text { edificio están bien conservados } \\
\text { (habitaciones, ascensores, pasillos } \\
\text { entre otros) }\end{array}$ \\
\hline $\begin{array}{l}\text { Dependencias e instalaciones deben } \\
\text { resultar agradables }\end{array}$ & $\begin{array}{l}\text { Dependencias e instalaciones } \\
\text { resultan agradables }\end{array}$ \\
\hline $\begin{array}{l}\text { Personal debe tener un aspecto limpio } \\
\text { y aseado }\end{array}$ & $\begin{array}{l}\text { Personal tiene un aspecto limpio y } \\
\text { aseado }\end{array}$ \\
\hline Instalaciones deben estar limpias & Instalaciones están limpias \\
\hline
\end{tabular}

Fuente: Elaboración propia

\section{Modelo LODGSERV}

LODGSERV, fue un modelo diseñado para medir las expectativas de los clientes en los establecimientos hoteleros. El estudio parte de la base de la calidad del servicio en la industria hotelera, el cual es difícil de medir debido a las propias características de los servicios y los hoteles en particular [23]. Es un índice de 26 indicadores diseñados para medir las expectativas de los clientes de los establecimientos hoteleros. Para realizar la investigación de este modelo, en principio se diseñó un cuestionario para evaluar la calidad de servicio (percepción menos expectativa) a partir del trabajo realizado por [6]. El cuestionario, posterior a los estudios preliminares, consistió en 26 preguntas. Los resultados confirman las cinco dimensiones sobre la calidad de servicio del modelo SERVQUAL. El orden de importancia, según la varianza experimentada por cada dimensión, es en primera instancia, los elementos relacionados con la fiabilidad, posterior a ello, los relacionados con la seguridad, seguidamente, se encuentra la capacidad de respuesta, en cuarto lugar, los elementos tangibles del establecimiento y por último la dimensión empatía [14].

\section{Modelo SERVPERF}

Tanto el modelo SERVPERF y SERVQUAL son los instrumentos para medir la calidad del servicio en diferentes tipos de empresas [26]. El modelo SERVPERF debe su nombre a la exclusiva atención que presta a la valoración del desempeño (SERVice PERFormance) para medir la calidad de servicio. Bajo este contexto, el SERVPERF se estructura de las mismas preguntas y dimensiones que el modelo SERVQUAL, sin embargo, la diferencia radica en la eliminación de la parte que hace mención a las expectativas de los clientes. Es decir, la escala SERVPERF tiene su base únicamente y exclusivamente en las percepciones, eliminando de esta manera las expectativas y reduciendo entonces a la mitad las preguntas planteadas [25].

Por esta razón, el razonamiento que fundamenta al método SERVPRF está directamente relacionado con los problemas de interpretación del concepto de expectativa, en su variabilidad en el transcurso de la prestación del servicio, y en su redundancia respecto a las percepciones [26].

Otras técnicas: cuestionarios de satisfacción del cliente 
Para recoger la opinión de los clientes, muchas organizaciones realizan encuestas, por ejemplo, aquellas que buscan medir la satisfacción.

Diseño de cuestionarios

Para tener una visión más general de la satis-facción del cliente resulta indispensable recopilar las opiniones sobre las funcionalidades especificas del producto o servicio que la organización ofrece, con la finalidad de relacionar las necesidades del cliente y la manera en que el producto satisface dichas necesidades [19].

Tabla 2. Evaluación especifica de un área funcional de una empresa de alojamiento

\begin{tabular}{|l|l|l|l|l|l|}
\hline \multicolumn{1}{|c|}{ Recepción } & Muy Bien & Bien & Regular & Mal & Muy Mal \\
\hline Amabilidad & & & & & \\
\hline Trato personal & & & & & \\
\hline
\end{tabular}

Fuente: Elaboración propia

Bajo este contexto, los cuestionarios que se elaboran para medir la calidad del servicio necesariamente se deben estructurar de la siguiente manera:

- La introducción, donde se explica el porqué de la encuesta y se inicia el proceso de recopilación de la información.

- El cuerpo principal, se debe cubrir la información general y específica sobre el producto y la empresa. Se puede anteponer una frase descriptiva en cada sección para que el cliente sea consciente de la existencia de un nuevo asunto.

- La conclusión, recopila la información personal del cliente y del servicio. Igualmente, se le puede agradecer su colaboración e indicar dónde puede entregar el cuestionario [17].

\section{Preguntas y respuestas}

Los clientes habitualmente tienen poco tiempo para contestar cuestionarios, es por ello que, la empresa que realiza esta actividad debe brindar facilidades para su realización. En consecuencia, los tipos de preguntas comúnmente utiliza-das constituyen las de valoración/evaluación.

De esta manera, las respuestas pueden ser de varios tipos:

- Abiertas: el cliente tiene un espacio en blanco donde puede exponer su opinión sobre lo que le están preguntando.

- Semicerradas: contienen una parte cerrada para que se seleccione la respuesta deseada pero también una parte abierta para añadir comentarios o bien contestar cuando la respuesta necesita información adicional o no se encuentra en la lista cerrada.

- Escala o secuencia continua: son aquellas en las que se solicita al cliente que asigne una puntuación a la pregunta según la importancia y experiencia del mismo. Se suele usar una escala denominada Likert, donde en un extremo se presenta una respuesta positiva y en el otro una negativa, con niveles intermedios [27]. 
Sistema de sugerencias y reclamaciones

Las empresas necesariamente deben desarrollar procedimientos que indiquen a sus empleados cómo comportarse y actuar en el caso que se presenten quejas y reclamos; dado que ofrecen otra oportunidad, y hay que estar preparados para no perderla. Debido a la dificultad de prever todas las posibles situaciones, es recomendable formar al personal de contacto en este aspecto a fin de resolver estos inconvenientes de forma correcta [28]. A partir, de aquello, a continuación, dentro de la figura 3 , se esquematiza el proceso de sugerencias y reclamaciones que una empresa de alojamiento debe realizar.

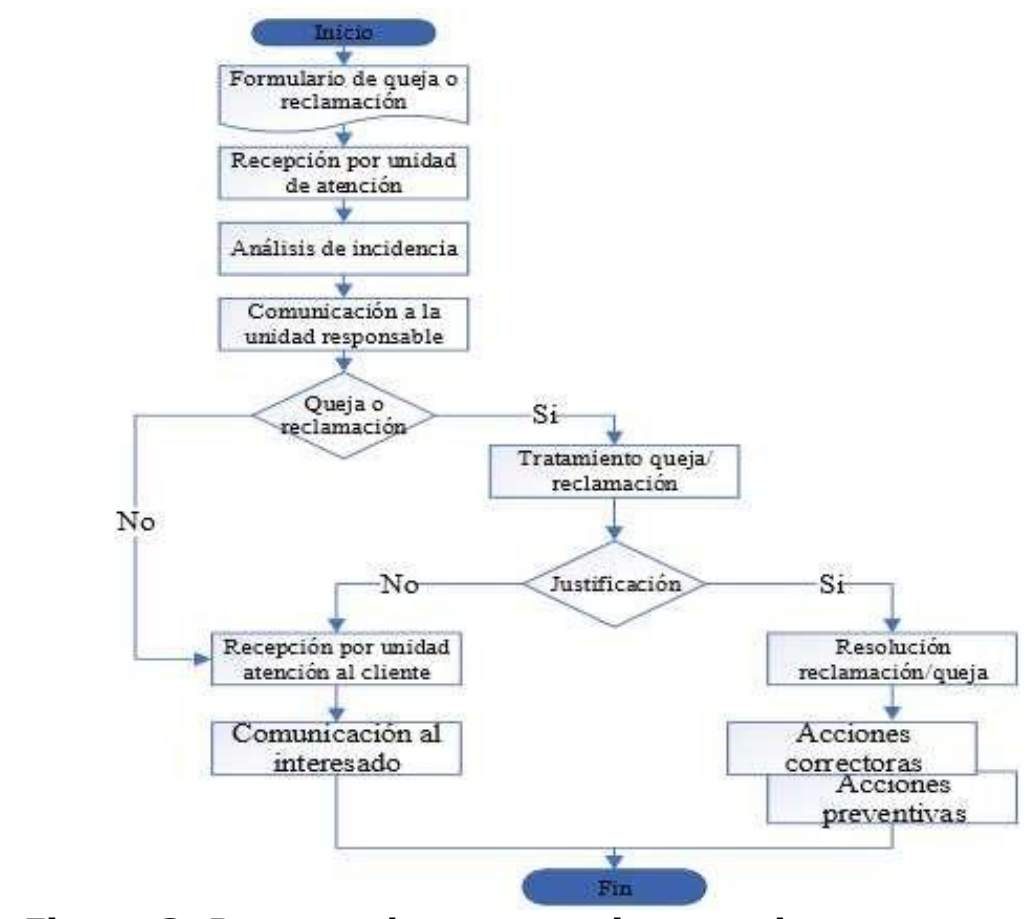

Figura 3. Proceso de sugerencias y reclamos

Técnica del cliente misterioso o Mistery Shoppers

Esta técnica del "cliente misterioso" es utilizada para el control de la calidad del servicio prestado, centrándose en el análisis del comportamiento del personal frente a situaciones reales en procesos de venta o a su vez de prestación de servicios. Su característica principal es porque un observador realiza el papel de cliente, realizando un proceso de compra y estableciendo en ciertas ocasiones situaciones extremas al personal de contacto para analizar su respuesta y la calidad del servicio prestado [29].

\section{Método}

El presente manuscrito se centra en la revisión de literatura, que engloba artículos científicos publicados en diferentes revistas a nivel mundial, buscando que las publicaciones sean las adecuadas sin restricción de idioma o año de publicación. Por otro lado, en la evaluación de la calidad de información obtenida tanto de medios científicos como de libros se analizaron aquellos que se enfocaron en la calidad de servicio y los métodos de medición en empresas hoteleras. A continuación, dentro de la tabla 3, se presentan los estudios considerados para la construcción del 
presente manuscrito.

\section{Tabla 3. Revisión de literatura}

\begin{tabular}{|c|c|c|c|c|c|}
\hline Scopus & Redalyc & EBSCO & Dialnet & Libros & $\begin{array}{l}\text { Proyectos de } \\
\text { investigación }\end{array}$ \\
\hline $\begin{array}{l}\text { "Towards an } \\
\text { understanding of total } \\
\text { service quality in hotels" }\end{array}$ & \multirow{2}{*}{$\begin{array}{l}\text { "Las } \\
\text { dimensiones de } \\
\text { la calidad det } \\
\text { servicio en el } \\
\text { proceso re de } \\
\text { distribución y } \\
\text { comercialización } \\
\text { de energia } \\
\text { eléctrica" }\end{array}$} & \multirow{2}{*}{$\begin{array}{l}\text { "Análisis de } \\
\text { los modelos } \\
\text { de medición } \\
\text { de calidad } \\
\text { percibida del } \\
\text { servicio } \\
\text { apticados en } \\
\text { la industria } \\
\text { de } \\
\text { hospitalidad" }\end{array}$} & \multirow{2}{*}{ 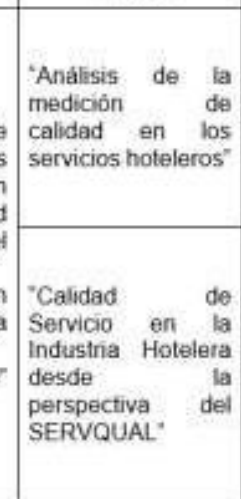 } & 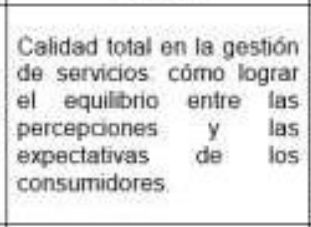 & $\begin{array}{l}\text { "Dimensiones } \\
\text { involucradas } \\
\text { en la } \\
\text { evaluación de } \\
\text { satisfacción } \\
\text { usuaria hacia } \\
\text { seguros de } \\
\text { salud en Chile" }\end{array}$ \\
\hline $\begin{array}{l}\text { "Measuring service quality } \\
\text { in the hotel industry. A } \\
\text { study in a business hotel } \\
\text { in Turkey" }\end{array}$ & & & & $\begin{array}{l}\text { Marketing de servicios. } \\
\text { personal, tecnologías y } \\
\text { estrategia. }\end{array}$ & $\begin{array}{l}\text { "El modelo } \\
\text { Servqual y su } \\
\text { incidencia en } \\
\text { el nivel de } \\
\text { satisfacción de } \\
\text { la carrera de } \\
\text { Economia de } \\
\text { la Universidad } \\
\text { Nacional De } \\
\text { Chimborazo" }\end{array}$ \\
\hline 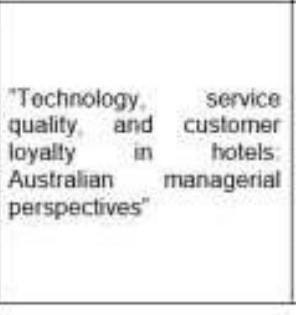 & 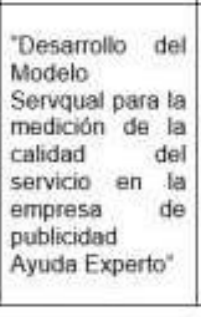 & & $\begin{array}{l}\text { 'Tunismo y calidad } \\
\text { del servicio' }\end{array}$ & $\begin{array}{l}\text { Gestión de la calidad de } \\
\text { los procesos turísticos } \\
\text { Procesos de gestión de } \\
\text { calidad en hosteleria y } \\
\text { turismo. }\end{array}$ & $\begin{array}{l}\text { Propuesta de } \\
\text { modelo de } \\
\text { medición de la } \\
\text { satistacción } \\
\text { del usuario en } \\
\text { una empresa } \\
\text { turistica Caso } \\
\text { Hotel } \\
\text { Valdivia's } \\
\text { Restaurant } \\
\text { Bar" }\end{array}$ \\
\hline $\begin{array}{l}\text { "Messuring Service } \\
\text { Quality Dimensions. An } \\
\text { Empirical Analysis of Thai } \\
\text { Hotel Industry" }\end{array}$ & & & $\begin{array}{lr}\text { "Modelos re de } \\
\text { gestión de la } \\
\text { calidad de servicio: } \\
\text { revisión r y } \\
\text { propuestar de } \\
\text { integración con la } \\
\text { estrategia } \\
\text { empresarial" }\end{array}$ & "Marketing" & \multirow{2}{*}{$\begin{array}{l}\text { "Elaboración } \\
\text { de un estudio } \\
\text { de carácter } \\
\text { exploratorio } \\
\text { para poner a } \\
\text { prueba una } \\
\text { herramienta de } \\
\text { identificación } \\
\text { de alternativas } \\
\text { de } \\
\text { recuperación } \\
\text { del servicio de } \\
\text { Hoteles de } \\
\text { Negocio" }\end{array}$} \\
\hline $\begin{array}{l}\text { "A Service Quality Model } \\
\text { Applied on Indian Hotel } \\
\text { Industry to Measure the } \\
\text { Level of Customer } \\
\text { Satisfaction" }\end{array}$ & & & $\begin{array}{l}\text { 'Análisis de la } \\
\text { Calidad } \\
\text { Servicio de lo los } \\
\text { Hoteles Pymes de } \\
\text { Bucaramanga y su } \\
\text { área } \\
\text { Metropolitana" }\end{array}$ & $\begin{array}{l}\text { "Propuesta de mejora de la } \\
\text { gestión de quejas y } \\
\text { reclamos en la empreșa } \\
\text { Cineplanet-Chiclayo" }\end{array}$ & \\
\hline $\begin{array}{l}\text { "Servqual Method applied } \\
\text { to Agencia Fiscal del } \\
\text { Estado de Sonors: An } \\
\text { analysis about service } \\
\text { quality" }\end{array}$ & & & $\begin{array}{l}\text { "Hotelqual: una } \\
\text { escala para medir } \\
\text { la calidad percibida } \\
\text { en servcios de } \\
\text { alojamiento" }\end{array}$ & $\begin{array}{l}\text { Procesos de gestión de } \\
\text { calidad en hosteleria y } \\
\text { turismo }\end{array}$ & \\
\hline $\begin{array}{l}\text { "Improving service } \\
\text { management in budget } \\
\text { hotels" }\end{array}$ & & & & & \\
\hline \multicolumn{6}{|l|}{$\begin{array}{l}\text { "A fuzzy SERVQUAL } \\
\text { based method for } \\
\text { evaluated of service } \\
\text { quality in the hotel } \\
\text { industry" }\end{array}$} \\
\hline $\begin{array}{l}\text { "Mystery shopping } \\
\text { indicadores de estudio en } \\
\text { bibliotecas" }\end{array}$ & & & & & \\
\hline
\end{tabular}

\begin{tabular}{|l|l|l|l|l|}
\hline $\begin{array}{l}\text { Hotel services quality in } \\
\text { developing countries } \\
\text { measured by SERVQUAL } \\
\text { modet }\end{array}$ & & & & \\
\hline
\end{tabular}




\section{Resultados}

De forma general, se pudo obtener información de 29 fuentes confiables y con una calidad de información muy buena para procesar la información a través de un proceso de lectura completa y un posterior análisis por parte de los investigadores.

Tal como se observa en la tabla 3, la información que se presenta en este estudio fue procesada de diferentes bases de datos (Scopus, Redalyc, EBSCO, Dialnet, Libros y Proyectos de investigación) de las cuales se detalló cada una de las categorías y subcategorías implícitas en la calidad de servicio y los métodos de medición de la misma que fueron utilizados en su debido tiempo en cada una de las investigaciones realizadas.

La revisión de todos los documentos citados en el apartado anterior muestra la importancia de la calidad del servicio para la adecuada gestión de una empresa, es por ello que, para tener un panorama más amplio, diferentes investigaciones han planteado diversos métodos que ayudan a medir este factor (calidad en el servicio), por ejemplo, dentro de esta amplia indagación se encontraron métodos tales como: HOTELQUAL, LOGSERV, SERVPERF, sin embargo, la base para el surgimiento de estos modelos lo constituyó el método SERVQUAL, el cual se encuentra estructurado por cinco dimensiones que buscan determinar el nivel de calidad en el servicio comparando expectativas vs percepciones.

\section{Conclusiones}

El presente manuscrito fue desarrollado tomando como referencia artículos que previamente abordaron temas similares al expuesto dentro de este estudio, en donde se encontró información variada, misma que fue procesada para la posterior construcción de la presente investigación, en ella se procesaron un total de 29 estudios, distribuidos de la siguiente manera: 19 artículos científicos, 6 libros y 4 proyectos de investigación que abordaron temas como la calidad del servicio y métodos de medición en el sector hotelero.

Este estudio ha contribuido al avance teórico de la literatura a través del análisis de algunas cuestiones fundamentales de los métodos para medir la calidad de servicio en empresas de alojamiento. Estableciendo con precisión la expectativa específica de los clientes, las dimensiones de la calidad del servicio en torno al cual los clientes realizan sus evaluaciones de calidad y su importancia para los clientes llevan vital importancia para los esfuerzos de mejora de la calidad.

Este estudio también ayuda en el descubrimiento de las necesidades, deseos y expectativas de los clientes. Estos son analizados mediante la determinación de las características de calidad de servicio que son más importantes para los clientes. Teniendo conocimiento de estas áreas ayudaría sin duda a los administradores a cumplir con el reto de mejorar la calidad del servicio en la industria hotelera.

El enfoque de los diferentes modelos estudiados constituye la medición de la calidad de los servicios, sin embargo, el método que es utilizado con mayor frecuencia es el SERVQUAL, el cual se encuentra estructura por cinco dimensiones (elementos tangibles, fiabilidad, capacidad de respuesta, seguridad, empatía), además constituye el punto de partida o de referencia para los demás modelos, comparando siempre expectativa vs percepción. 
La calidad del servicio constituye el factor decisorio para determinar la satisfacción del cliente en los centros de alojamiento, por lo cual resulta importante conocer que los componentes de calidad de servicio son los impulsores de rendimiento del establecimiento. Es decir, que los componentes de calidad de servicio se traducen en las operaciones que a diario realiza el centro de alojamiento, que puede ser manipulado por la dirección.

\section{Referencias bibliográficas}

[1] H. Wilkins, B. Merrilees and C. Herington, "Towards an understanding of total service quality in hotels," International Journal of Hospitality Management, vol. 26, pp. 840-853, 2007.

[2] A. Akbaba, "Measuring service quality in the hotel industry: A study in a business hotel in Turkey," International Journal of Hospitality Management, vol. 25, no. 2, pp. 170-192, 2010.

[3] S. C. Lee, S. Barker, and J. Kandampully, "Technology, service quality, and customer loyalty in hotels: Australian managerial perspectives," Manag. Serv. Qual. An Int. J., vol. 13, no. 5, pp. 423-432, 2013.

[4] S. Boon itt and N. Rompho, "Measuring Service Quality Dimensions: An Empirical Analysis of Thai Hotel Industry," Int. J. Bus. Adm., vol. 3, no. 5, pp. 52-63, 2012.

[5] Sharma, "A Service Quality Model Applied on Indian Hotel Industry to Measure the Level of Customer Satisfaction," Int. J. Sci. Res., vol. 3, no. 3, pp. 480-485, 2014.

[6] V. Zeithaml, L. Berry, and A. Parasuraman, Calidad total en la gestión de servicios: cómo lograr el equilibrio entre las percepciones y las expectativas de los consumidores. Madrid, 1992.

[7] L. Ibarra, E. Casas, and A. Partida, "Servqual Method applied to Agencia Fiscal del Estado de Sonora: An analysis about service quality," Procedia - Soc. Behav. Sci., vol. 148, pp. 87-93, 2014.

[8] J. Peng, X. Zhao, and A. Mattila, "Improving service management in budget hotels," International Journal of Hospitality Management, vol. 49, pp. 139-148, 2015.

[9] ]. M. Daza Higuera, "Análisis de la medición de calidad en los servicios hoteleros," Revista Criterio Libre, vol. 11, no. 19, pp. 263-280, 2013.

[10] N. Stefano, N. Casarotto Filho, R. Barichello, and A. Sohn, "A fuzzy SERVQUAL based method for evaluated of service quality in the hotel industry," Procedia CIRP, vol. 30, pp. 433-438, 2015.

[11] Lovelock and J. Wirtz, Marketing de servicios: personal, tecnologías y estrategia, Sexta Edic. México D.F., 2009.

[12] M. I. Vergara Henríquez, "Dimensiones involucradas en la evaluación de satisfacción usuaria hacia seguros de salud en Chile," Universidad de Chile, 2014.

[13] M. E. Dávila Bustamante, J. Á. Coronado Quintana, and B. M. Cerecer Castro, "Las dimensiones de la calidad del servicio en el proceso de distribución y 
comercialización de energía eléctrica," Contaduría y Adm., vol. 57, no. 3, pp. 175-195, 2012.

[14] J. Ríos and R. Santomá, "Calidad de Servicio en la Industria Hotelera desde la perspectiva del SERVQUAL," Manag. Empres., pp. 1-12, 2008.

[15] De la Torre Tejada, "Turismo y calidad del servicio," Cultura, vol. 25, pp. 127-142, 2011.

[16] Serrano and M. C. López Fernández, "Modelos de gestión de la calidad de servicio: revisión y propuesta de integración con la estrategia empresarial," ResearchGate, vol. 1, pp. 1-10, 2014.

[17] M. Alonso Almeida, L. Barcos Redín, and J. I. Martín Castilla, Gestión de la calidad de los procesos turísticos. Madrid, 2006.

[18] S. Carrasco Fernández, Procesos de gestión de calidad en hostelería y turismo. Madrid, 2013.

[19] Monsalve Castro and S. I. Hernández Rueda, "Análisis de la Calidad del Servicio de los Hoteles Pymes de Bucaramanga y su área Metropolitana," Sotavento M.B.A., vol. 26, pp. 62-69, 2015.

[20] Falces Delgado, B. Sierra Díez, A. Becerra Grande, and P. Briñol Turnes, "Hotelqual: una escala para medir la calidad percibida en servicios de alojamiento," Estud. turísticos, no. 139, pp. 93-108, 1999.

[21] M. González Bautista, E. Zurita Moreno, and M. Zurita Vaca, "El modelo Servqual y su incidencia en el nivel de satisfacción de la carrera de Economía de la Universidad Nacional De Chimborazo," Eur. Sci. J., vol. 13, no. 25, pp. 339352, 2017.

[22] R. Matsumoto Nishizawa, "Desarrollo del Modelo Servqual para la medición de la calidad del servicio en la empresa de publicidad Ayuda Experto," Perspectivas, no. 34, pp. 181-209, 2014.

[23] J. Duque Oliva and M. C. Parra Díaz, "Análisis de los modelos de medición de calidad percibida del servicio aplicados en la industria de hospitalidad," Perspect. Empres., vol. 2, no. 2, pp. 35-54, 2015.

[24] Besáñez Dávalos, "Propuesta de modelo de medición de la satisfacción del usuario en una empresa turística: Caso Hotel Valdivia's Restaurant Bar," Universidad Autónoma de Aguascalientes, 2017.

[25] L. F. Escobar Velasco and V. Roa Giraldo, "Elaboración de un estudio de carácter exploratorio para poner a prueba una herramienta de identificación de alternativas de recuperación del servicio de Hoteles de Negocio," Universidad ICESI, 2014.

[26] V. Babic Hodovic, M. Arslanagié Kalajdzic, and A. Banda, "Hotel services quality in developing countries measured by SERVQUAL model," Acta Geogr. Bosniae Herzegovinae, vol. 4, pp. 5-24, 2015.

[27] AU Consultores, "Marketing," Madrid, 2013.

[28] S. B. Guerrero Rosillo and F. de J. Trujillo Perea, "Propuesta de mejora de la gestión de quejas y reclamos en la empresa Cineplanet-Chiclayo," Universidad Católica Santo Toribio de Mogrovejo, 2014. 


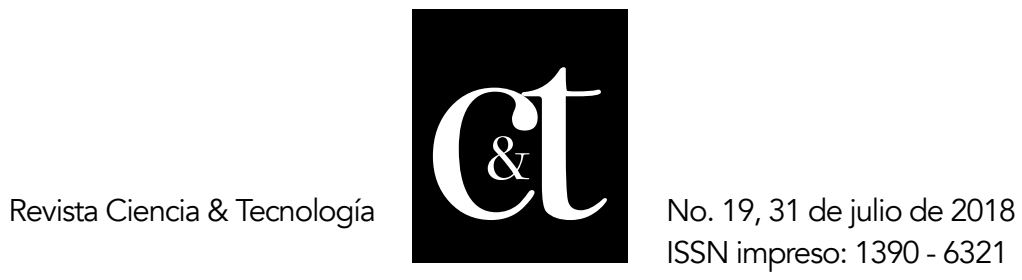

[29] Morena de Diago, "Mystery shopping: indicadores de estudio en bibliotecas," Rev. Gen. Inf. y Doc., vol. 23, no. 2, pp. 369-386, 2013. 